\title{
HIGHER-ORDER ESTIMATES FOR FULLY NONLINEAR DIFFERENCE EQUATIONS. II
}

\author{
DEREK W. HOLTBY \\ CSIRO Telecommunications and Industrial Physics, \\ Epping, NSW 1710, Australia
}

(Received 2 March 1998)

\begin{abstract}
The purpose of this work is to establish a priori $C^{2, \alpha}$ estimates for mesh function solutions of nonlinear difference equations of positive type in fully nonlinear form on a uniform mesh, where the fully nonlinear finite difference operator $\mathcal{F}_{h}$ is concave in the second-order variables. The estimate is an analogue of the corresponding estimate for solutions of concave fully nonlinear elliptic partial differential equations. We use the results for the special case that the operator does not depend explicitly upon the independent variables (the so-called frozen case) established in part I to approach the general case of explicit dependence upon the independent variables. We make our approach for the diagonal case via a discretization of the approach of Safonov for fully nonlinear elliptic partial differential equations using the discrete linear theory of Kuo and Trudinger and an especially agreeable mesh function interpolant provided by Kunkle. We generalize to non-diagonal operators using an idea which, to the author's knowledge, is novel. In this paper we establish the desired Hölder estimate in the large, that is, on the entire mesh $n$-plane. In a subsequent paper a truly interior estimate will be established in a mesh $n$-box.
\end{abstract}

Keywords: fully nonlinear difference equations; discrete a priori Hölder estimates; discrete seminorms; non-local discrete Hessian

AMS 2000 Mathematics subject classification: Primary 35J60; 35J15; 39A12

Secondary 39A70; 39A10; 65N06; 65N22; 65N12

\section{Introduction}

In this paper the setting and notation are as in [6], where we derived a discrete a priori $C^{2, \alpha}$ estimate for solutions of difference equations involving operators of the form

$$
F_{h}[u](x) \equiv \mathcal{F}_{h}\left(\delta^{2} u(x)\right) .
$$

Here our purpose is to use that estimate to derive a discrete a priori $C^{2, \alpha}$ estimate for solutions of the problem

$$
F_{h}[u](x)=0, \quad \forall x \in \mathbb{Z}_{h}^{n},
$$

for more general difference operators

$$
F_{h}[u](x) \equiv \mathcal{F}_{h}\left(x, \delta^{2} u(x)\right),
$$


where, with $Y_{N}=\left\{y \in \mathbb{Z}_{h}^{n} \backslash\{0\}:\|y\|_{\infty} \leqslant N h\right\}$,

$$
\delta^{2} u(x)=\left\{\delta_{y}^{2} u(x) \mid y \in Y_{N}\right\},
$$

and $\mathcal{F}_{h}$ is a given real-valued function on $\Gamma=\mathbb{R}^{n} \times \mathbb{R}^{Y_{N}}$. We do not address the question of existence in this paper.

Denote points of $\Gamma$ by ordered pairs $(x, s)$. We assume that $\mathcal{F}_{h}$ is differentiable in $s_{y}$ for each $y \in Y_{N}$ and symmetric with respect to $s_{ \pm y}$ for each $y \in Y_{N}$. We assume that there is $Y^{\prime} \subset Y_{N}$ such that $\left\{h e_{1}, \ldots, h e_{n}\right\} \subset Y^{\prime}$, and, for all $(x, s) \in \Gamma, \mathcal{F}_{h}$ satisfies

$$
0<\lambda \leqslant \frac{\partial \mathcal{F}_{h}(x, s)}{\partial s_{y}} \leqslant \Lambda \quad \text { for all } y \in Y^{\prime}, \quad \text { and } \quad \frac{\partial \mathcal{F}_{h}(x, s)}{\partial s_{y}} \equiv 0 \quad \text { for all } y \in Y_{N} \backslash Y^{\prime} \text {, }
$$

for some positive constants $\lambda, \Lambda$. By the symmetry above, $y \in Y^{\prime}$ if and only if $-y \in Y^{\prime}$. Again, our major assumption in addition to the above is that $\mathcal{F}_{h}$ is concave in the second-order variables $\left\{s_{y}\right\}$.

The only such estimate known to the author is for the two-dimensional case by Hackbusch in [4].

As in the continuous case (see $[\mathbf{1 1}, \mathbf{1 2}]$ ), we derive our estimate by viewing (1.1) locally as a perturbation in $x$ of an equation of the same form but with $x$ fixed.

As in $[\mathbf{6}]$, in order to achieve our goal we rely upon the existence of a special interpolant of mesh functions, provided by Kunkle [8] (generalizes [2] and [1] to $n$-dimensional realvalued mesh functions) (see $[\mathbf{6}, \S 4]$ ). Let us restate [6, Theorem 4.1], augmenting it somewhat.

Again we denote the set of points used in the definition of a difference quotient by supp, the support of the difference quotient, and for any set of mesh points $S \subset \mathbb{Z}_{h}^{n}$ we define

$$
\left|\delta^{\alpha} u\right|_{0 ; S}=\max _{x \in S, \operatorname{supp} \delta^{\alpha} u(x) \subset S}\left|\delta^{\alpha} u(x)\right| .
$$

Lemma 1.1 (Theorem 13.2 in [7]). Let $\Omega$ be the Cartesian product of $n$ closed (not necessarily finite) intervals such that the corners of $\Omega$ are themselves lattice points, and assume that in each of the coordinate directions $e_{j}, \Omega$ has diameter at least $3 h$. With $\Omega_{h}=\Omega \cap \mathbb{Z}_{h}^{n}$, let $u: \Omega_{h} \rightarrow \mathbb{R}$ be a mesh function on $\Omega_{h}$. Then there exists an interpolant $u_{\mathrm{e}}=F^{h} u \in C^{\infty}\left(\mathbb{R}^{n}\right)$ (so $u_{\mathrm{e}}(x)=u(x)$ for all mesh points $x \in \Omega_{h}$ ) and a constant $C$ depending only upon $n$ and the choice of a particular compactly supported function $\psi: \mathbb{R} \rightarrow \mathbb{R}$ (we fix a choice for the duration of this paper, thus rendering later definitions of semi-norms unambiguous) such that for all multi-indices $\beta$ satisfying $0 \leqslant \beta_{i} \leqslant 3$ for each $i=1, \ldots, n$, we have

$$
\left|D^{\beta} u_{\mathrm{e}}\right|_{0 ; \Omega} \leqslant C\left|\delta^{\beta} u\right|_{0 ; \Omega_{h}} .
$$

In fact, if $z \in \Omega_{h}$ and $\operatorname{dist}(z, \partial \Omega) \geqslant 2 h$, then

$$
\left|D^{\beta} u_{\mathrm{e}}\right|_{0 ; z+[0, h]^{n}} \leqslant C\left|\delta^{\beta} u\right|_{0 ; z+[-h, 2 h]^{n} \cap \mathbb{Z}_{h}^{n}} .
$$

If $\delta^{\beta} u$ is not bounded (for instance if $\Omega$ is not compact), then for every compact subset $K$ of $\Omega$ there is a compact $K^{\prime} \subset \Omega$ such that

$$
\left|D^{\beta} u_{\mathrm{e}}\right|_{0 ; K} \leqslant C\left|\delta^{\beta} u\right|_{0 ; K^{\prime} \cap \mathbb{Z}_{h}^{n}} .
$$


Furthermore, for all multi-indices $\beta$ satisfying $\|\beta\|_{1}=3$ :

$$
\left|D^{\beta} u_{\mathrm{e}}\right|_{0 ; \Omega} \leqslant C \sup _{1 \leqslant i \leqslant n, w \in Y_{1}}\left|\delta_{h e_{i}}^{+} \delta_{w}^{2} u\right|_{0 ; \Omega_{h}} .
$$

Again, if $z \in \Omega_{h}$ and $\operatorname{dist}(z, \partial \Omega) \geqslant 2 h$, then

$$
\left|D^{\beta} u_{\mathrm{e}}\right|_{0 ; z+[0, h]^{n}} \leqslant C \sup _{1 \leqslant i \leqslant n, w \in Y_{1}}\left|\delta_{h e_{i}}^{+} \delta_{w}^{2} u\right|_{0 ; z+[-h, 2 h]^{n} \cap \mathbb{Z}_{h}^{n}} .
$$

We still have, of course, that for fixed $x \in \Omega$ such that $\operatorname{dist}(x, \partial \Omega) \geqslant 2 h, F^{h} u(x)$ is independent of $u(z)$ for $z \in \mathbb{Z}_{h}^{n}$ when $\|z-x\|_{\infty}>2 h$.

The operator $F^{h}$ is linear, and it reproduces polynomials of maximum degree 2 in each variable in the sense that if $u$ is the restriction to the mesh of such a polynomial, then $F^{h} u$ is that polynomial. The Taylor polynomial of total degree at most two of the extension about a mesh point agrees with the extension at orthogonally adjacent mesh points. That is, if $T_{\bar{x}, 2} u_{\mathrm{e}}$ is the said Taylor polynomial of the extension $u_{\mathrm{e}}$ (of $u$ ) about $\bar{x}$, where $\bar{x} \in \mathbb{Z}_{h}^{n}$ is a mesh point, then for any $i \in\{1,2, \ldots, n\}, T_{\bar{x}, 2} u_{\mathrm{e}}\left(\bar{x} \pm h e_{i}\right)=u_{\mathrm{e}}\left(\bar{x} \pm h e_{i}\right)$. It follows that the centred second-order coordinate-direction difference quotients of $T_{\bar{x}, 2} u_{\mathrm{e}}(x)$ (which are constant) coincide with the corresponding difference quotients of the mesh function $u$ at $\bar{x}$.

This results from a straightforward modification of Kunkle's [7] result. (See [5] for an exposition of Kunkle's construction and details of the said modification, as well as proofs of the facts contained in the above lemma.)

The restriction to the form $\delta_{h e_{i}}^{+} \delta_{w}^{2} u, w \in Y_{1}$, in the above lemma is very useful, because we can relate such terms to $C^{2, \alpha}$ estimates via the following manipulation:

$$
\delta_{h e_{i}}^{+} \delta_{w}^{2} u(x)=\frac{\delta_{w}^{2} u\left(x+h e_{i}\right)-\delta_{w}^{2} u(x)}{h}=\frac{\delta_{w}^{2} u\left(x+h e_{i}\right)-\delta_{w}^{2} u(x)}{h^{\alpha}} h^{\alpha-1} .
$$

Our goal is to prove Theorem 4.1. We prove this theorem first in the case of diagonal operators by discretizing Safonov's derivation of a Hölder estimate for second derivatives of solutions of concave fully nonlinear elliptic partial differential equations (see $[\mathbf{1 1}]$ and [12]). Kunkle's extension facilitates the application of technical calculus lemmas from Safonov's work. We establish (in $\S 2$ ) that on $\mathbb{Z}_{h}^{n}$ a mesh function $u$ and its extension $u_{\mathrm{e}}$ of Lemma 1.1 have equivalent $C^{2, \alpha}$ Hölder norms. We prove this equivalence non-globally as well, for a mesh function defined on an $n$-rectangle. We will then follow Safonov's proof, moving to or from a Hölder semi-norm of an a priori solution of (4.1) from or to the corresponding Hölder semi-norm of the extension by equivalence of semi-norms as is convenient; that is, when we wish to use information from the difference equation (4.1) we move by equivalence of semi-norms to the discrete Hölder semi-norm of the mesh function. When we wish to use calculus-based technical lemmas from Safonov's work we move by equivalence of semi-norms to the Hölder semi-norm of the extension of the mesh solution. We generalize the proof to non-diagonal operators using an idea which, to the author's knowledge, is novel. 


\section{Semi-norms}

Here we add to the definitions in $[\mathbf{6}, \S 1.1]$. With the exception of the semi-norms on the left in (2.1), the definitions in this section are trivial modifications of definitions in $[\mathbf{1 1}, \mathbf{1 2}]$. Let $\Omega$ be a domain (open or closed) in $\mathbb{R}^{n}$, and $u: \Omega \rightarrow \mathbb{R}$. For $m$ a nonnegative integer and $0<\alpha<1$, we set

$$
\left.\begin{array}{rlrl}
{[u]_{0, \alpha ; \Omega}} & =\sup _{x, y \in \Omega, x \neq y} \frac{|u(x)-u(y)|}{\|x-y\|_{2}^{\alpha}}, & \langle u\rangle_{\Omega}^{(\alpha)} & =\sup _{x \in \Omega, \rho>0} \rho^{-\alpha} \underset{\Omega_{\rho}(x)}{\operatorname{osc}} u, \\
{[u]_{m, \alpha ; \Omega}} & =\max _{\|\beta\|_{1}=m}\left[D^{\beta} u\right]_{0, \alpha ; \Omega}, & \langle u\rangle_{\Omega}^{(m+\alpha)} & =\max _{\|\beta\|_{1}=m}\left\langle D^{\beta} u\right\rangle_{\Omega}^{(\alpha)},
\end{array}\right\}
$$

where $\operatorname{osc}_{\Omega_{\rho}(x)} u=\sup _{y, z \in \Omega_{\rho}(x)}|u(y)-u(z)|$ and $\Omega_{\rho}(x)=\Omega \cap K_{\rho}(x)$. Recall that $K_{\rho}(z)$ is the closed cube, parallel to the coordinate axes, with centre $z$ and side length $2 \rho$ : $K_{\rho}(z)=\left\{x \in \mathbb{R}^{n}:\|x-z\|_{\infty} \leqslant \rho\right\}$. The semi-norms on the left in (2.1) are equivalent, respectively, to those on the right, the constant of equivalence being dependent only upon $n$ since $0<\alpha<1$.

Let $\mathcal{P}_{m}$, for non-negative integer $m$, denote the collection of all $m$ th-degree polynomials

$$
p(x)=\sum_{\|\beta\|_{1} \leqslant m} C_{\beta} x^{\beta}, \quad C_{\beta}=\text { const. }
$$

where $\beta$ is a multi-index, and $x^{\beta}=\prod_{i} x_{i}^{\beta_{i}}$. Then, for example, the Taylor polynomial of total degree at most $m$ for the function $u$ at the point $x$ is

$$
T_{x, m} u(y)=\sum_{\|\beta\|_{1} \leqslant m} \frac{D^{\beta} u(x)}{\beta}(y-x)^{\beta} \in \mathcal{P}_{m} .
$$

We define the following:

$$
\begin{aligned}
& \omega_{m}(u, V)=\max _{\|\beta\|_{1}=m} \operatorname{osc} D^{\beta} u ; \\
& E_{m}[u ; V]=\inf _{p \in \mathcal{P}_{m}} \sup _{x \in V}|u(x)-p(x)| .
\end{aligned}
$$

If a function $v: \mathbb{R}^{n} \rightarrow \mathbb{R}$ has finite semi-norm $\langle v\rangle_{\mathbb{R}^{n}}^{(m+\alpha)}$, then this semi-norm is equivalent to the semi-norm

$$
\mu_{m+\alpha}^{*}(v)=\sup _{x \in \mathbb{R}^{n}, \rho>0} \rho^{-m-\alpha} E_{m}\left[v ; K_{\rho}(x)\right],
$$

a Campanato semi-norm $[\mathbf{1 1}, \mathbf{1 2}]$; that is, for some constant $C=C(n, m, \alpha)>0$ we have

$$
C^{-1}\langle v\rangle_{\mathbb{R}^{n}}^{(m+\alpha)} \leqslant \mu_{m+\alpha}^{*}(v) \leqslant C\langle v\rangle_{\mathbb{R}^{n}}^{(m+\alpha)} .
$$

This is proved in $\left[\mathbf{1 2}\right.$, Theorem $\left.2.1^{\prime}\right]$. Observe that with the notation introduced above, we may write the semi-norm $\langle v\rangle_{\mathbb{R}^{n}}^{(m+\alpha)}$ as

$$
\langle v\rangle_{\mathbb{R}^{n}}^{(m+\alpha)}=\sup _{\rho>0, x \in \mathbb{R}^{n}} \rho^{-\alpha} \omega_{m}\left(v, K_{\rho}(x)\right) .
$$


Let $u: \mathbb{Z}_{h}^{n} \rightarrow \mathbb{R}$ be a mesh function and let $u_{\mathrm{e}}: \mathbb{R}^{n} \rightarrow \mathbb{R}$ be the extension of $u$ in Lemma 1.1. We define the quantities

$$
\begin{aligned}
& \underline{\mu}_{2+\alpha}(u)=\sup _{\rho \geqslant h, x \in \mathbb{R}^{n}} \rho^{-\alpha} \omega_{2}\left(u_{\mathrm{e}}, K_{\rho}(x)\right), \\
& \underline{\mu}_{2+\alpha}^{*}(u)=\sup _{\rho \geqslant h, x \in \mathbb{R}^{n}} \rho^{-2-\alpha} E_{2}\left[u_{\mathrm{e}} ; K_{\rho}(x)\right] .
\end{aligned}
$$

We also extend the domain of the semi-norms $\langle\cdot\rangle_{\mathbb{R}^{n}}^{(2+\alpha)}$ and $\mu_{2+\alpha}^{*}(\cdot)$ to include mesh functions by defining

$$
\langle u\rangle_{\mathbb{R}^{n}}^{(2+\alpha)}=\left\langle u_{\mathrm{e}}\right\rangle_{\mathbb{R}^{n}}^{(2+\alpha)}, \quad \mu_{2+\alpha}^{*}(u)=\mu_{2+\alpha}^{*}\left(u_{\mathrm{e}}\right) .
$$

\section{Semi-norm equivalences}

The first lemma gives some well-known interpolation inequalities in terms of Safonov's semi-norms. The proof is almost identical to the proof of [3, Lemma $6.32, \S 6.8]$, but is made easier by virtue of being in an $n$-cube rather than a ball.

Lemma 3.1 (Lemma 2.1 in [12]). If $x \in \mathbb{R}^{n}, \rho>0,0<k+\alpha^{\prime}<m+\alpha(k$ and $m$ non-negative integers; $\left.0 \leqslant \alpha, \alpha^{\prime} \leqslant 1\right)$, and $u \in C^{m, \alpha}\left(K_{\rho}(x)\right)$, then

$$
\rho^{k+\alpha^{\prime}}\langle u\rangle_{K_{\rho}(x)}^{\left(k+\alpha^{\prime}\right)} \leqslant \varepsilon \rho^{m+\alpha}\langle u\rangle_{K_{\rho}(x)}^{(m+\alpha)}+C\left(\varepsilon, n, k, m, \alpha, \alpha^{\prime}\right) \sup _{K_{\rho}(x)}|u|,
$$

for all $\varepsilon>0$.

A consequence is the following lemma.

Lemma 3.2 (Corollary 2.2 in [12]). If $x \in \mathbb{R}^{n}, \rho>0, m$ a non-negative integer, $0 \leqslant \alpha \leqslant 1$, and $u \in C^{m, \alpha}\left(K_{\rho}(x)\right)$, then

$$
\rho^{m} \omega_{m}\left(u, K_{\rho}(x)\right) \leqslant \varepsilon \rho^{m+\alpha}\langle u\rangle_{K_{\rho}(x)}^{(m+\alpha)}+C(\varepsilon, n, m, \alpha) E_{m}\left[u ; K_{\rho}(x)\right],
$$

for all $\varepsilon>0$.

The following lemma is Lemma 2.2 of [12] stated for cubes rather than for balls. It is another consequence of Lemma 3.1 and the fact that for arbitrary $p \in \mathcal{P}_{m}$ we have $\langle u-p\rangle^{(m+\alpha)}=\langle u\rangle^{(m+\alpha)}$.

Lemma 3.3 (modification for cubes of Lemma 2.2 in [12]). If $x \in \mathbb{R}^{n}, \rho>0$, $m$ a non-negative integer, and $u \in C^{m, \alpha}\left(K_{\rho}(x)\right)$, where $0 \leqslant \alpha<1$, or $u \in C^{m+1}\left(K_{\rho}(x)\right)$, where $\alpha=1$, then

$$
\sup _{K_{\rho}(x)}\left|u-T_{x, m} u\right| \leqslant C(n) \rho^{m+\alpha}\langle u\rangle_{K_{\rho}(x)}^{(m+\alpha)},
$$

where $T_{x, m} u$ is the Taylor polynomial defined in (2.2).

We may now prove the following theorem wherein continuous semi-norm of interpolant meets discrete semi-norm of mesh function via equivalence. 
Theorem 3.4. Suppose $0<\alpha<1$. Let $\Omega$ be the Cartesian product of $n$ closed (not necessarily finite) intervals such that the corners of $\Omega$ are themselves lattice points, and assume that in each of the coordinate directions $e_{j}, \Omega$ has diameter at least $3 h$. With $\Omega_{h}=\Omega \cap \mathbb{Z}_{h}^{n}$, let $u: \Omega_{h} \rightarrow \mathbb{R}$ be a real-valued mesh function on $\Omega_{h}$ and let $u_{\mathrm{e}} \in C^{\infty}\left(\mathbb{R}^{n}\right)$ be the Kunkle extension of $u$ to $\mathbb{R}^{n}$, as in Lemma 1.1. There exist positive constants $C=C(n, N)$ and $D=D(n)$ such that

$$
C_{N}[u]_{2, \alpha ; \Omega_{h}} \leqslant\left[u_{\mathrm{e}}\right]_{2, \alpha ; \Omega} \leqslant D_{1}[u]_{2, \alpha ; \Omega_{h}},
$$

when one of these quantities is finite.

Proof. We prove the left-hand inequality first. Recall from $[\mathbf{6}, \S 1.1]$ that

$$
{ }_{N}[u]_{2, \alpha ; \Omega_{h}}=\sup _{\substack{x, y \in \Omega_{h}, x \neq y, z \in Y_{N} \\ x \pm z, y \pm z \in \Omega_{h}}} \frac{\left|\delta_{z}^{2} u(x)-\delta_{z}^{2} u(y)\right|}{\|x-y\|_{2}^{\alpha}} .
$$

Choose distinct $x, y \in \Omega_{h}$ and $z \in Y_{N}$ such that $x \pm z, y \pm z \in \Omega_{h}$. By the mean value theorem we have

$$
\begin{aligned}
\frac{\left|\delta_{z}^{2} u(x)-\delta_{z}^{2} u(y)\right|}{\|x-y\|_{2}^{\alpha}} & =\frac{\left|D_{\hat{z} \hat{z}} u_{\mathrm{e}}\left(x^{\prime}\right)-D_{\hat{z} \hat{z}} u_{\mathrm{e}}\left(y^{\prime}\right)\right|}{\|x-y\|_{2}^{\alpha}} \\
& \leqslant n \frac{\left|D^{\beta} u_{\mathrm{e}}\left(x^{\prime}\right)-D^{\beta} u_{\mathrm{e}}\left(y^{\prime}\right)\right|}{\|x-y\|_{2}^{\alpha}},
\end{aligned}
$$

where $\hat{z}=z /\|z\|_{2}, x^{\prime}=x+t z$ for some $t \in(-1,1), y^{\prime}=y+s z$ for some $s \in(-1,1)$, and where $\|\beta\|_{1}=2$ gives the maximum of $\left|D^{\beta} u_{\mathrm{e}}\left(x^{\prime}\right)-D^{\beta} u_{\mathrm{e}}\left(y^{\prime}\right)\right|$ over all such multiindices. It follows that $\left\|x^{\prime}-x\right\|_{2} \leqslant N \sqrt{n} h$ and $\left\|y^{\prime}-y\right\|_{2} \leqslant N \sqrt{n} h$. Therefore, if $x^{\prime} \neq y^{\prime}$ (if $x^{\prime}=y^{\prime}$, then $\delta_{z}^{2} u(x)=\delta_{z}^{2} u(y)$ ), then

$$
\frac{\left\|x^{\prime}-y^{\prime}\right\|_{2}}{\|x-y\|_{2}} \leqslant 2 N \sqrt{n}+1
$$

by the triangle inequality. Hence,

$$
\begin{aligned}
\frac{\left|\delta_{z}^{2} u(x)-\delta_{z}^{2} u(y)\right|}{\|x-y\|_{2}^{\alpha}} & \leqslant n(2 N \sqrt{n}+1)^{\alpha} \frac{\left|D^{\beta} u_{\mathrm{e}}\left(x^{\prime}\right)-D^{\beta} u_{\mathrm{e}}\left(y^{\prime}\right)\right|}{\left\|x^{\prime}-y^{\prime}\right\|_{2}^{\alpha}} \\
& \leqslant n(2 N \sqrt{n}+1)\left[u_{\mathrm{e}}\right]_{2, \alpha ; \Omega} .
\end{aligned}
$$

Taking the supremum over distinct $x, y \in \Omega_{h}, z \in Y_{N}$, we have

$$
{ }_{N}[u]_{2, \alpha ; \Omega_{h}} \leqslant n(2 N \sqrt{n}+1)\left[u_{\mathrm{e}}\right]_{2, \alpha ; \Omega} .
$$

For the right-hand inequality the proof is similar to the proof of Lemma 4.3 of [6]. Choose distinct $x, y \in \Omega$ and multi-index $\beta$ such that $\|\beta\|_{1}=2$. Consider two cases: (i) $\|x-y\|_{\infty} \leqslant h$ and (ii) $\|x-y\|_{\infty}>h$. 
Case (i): $\|\boldsymbol{x}-\boldsymbol{y}\|_{\infty} \leqslant \boldsymbol{h}$. With $\hat{\eta}=(x-y) /\|x-y\|_{2}$ we have, by the mean value theorem, for some $t \in(0,1), \xi=t x+(1-t) y$, that

$$
\frac{\left|D^{\beta} u_{\mathrm{e}}(x)-D^{\beta} u_{\mathrm{e}}(y)\right|}{\|x-y\|_{2}^{\alpha}}=\left|D_{\hat{\eta}} D^{\beta} u_{\mathrm{e}}(\xi)\right|\|x-y\|_{2}^{1-\alpha} \leqslant\left|D_{\hat{\eta}} D^{\beta} u_{\mathrm{e}}(\xi)\right|(\sqrt{n} h)^{1-\alpha} .
$$

Then by (1.2), Lemma 1.1, there exists $z \in \Omega_{h}, v \in\left\{h e_{1}, \ldots, h e_{n}\right\}$, and $w \in Y_{1}$ with $\operatorname{supp} \delta_{v}^{+} \delta_{w}^{2} u(z) \subset \Omega_{h}$ such that

$$
\left|D_{\hat{\eta}} D^{\beta} u_{\mathrm{e}}(\xi)\right| \leqslant C(n)\left|\delta_{v}^{+} \delta_{w}^{2} u(z)\right|,
$$

and, hence,

$$
\frac{\left|D^{\beta} u_{\mathrm{e}}(x)-D^{\beta} u_{\mathrm{e}}(y)\right|}{\|x-y\|_{2}^{\alpha}} \leqslant C \sqrt{n}_{1}[u]_{2, \alpha ; \Omega_{h}} .
$$

Case (ii): $\|\boldsymbol{x}-\boldsymbol{y}\|_{\infty}>\boldsymbol{h}$. Choose one of the mesh points nearest to $x$ (if $x$ is not a mesh point); that is, we choose $x_{h} \in \Omega_{h}$ such that $0 \leqslant\left\|x_{h}-x\right\|_{\infty}<h$. Likewise choose $y_{h} \in \Omega_{h}$ such that $0 \leqslant\left\|y_{h}-y\right\|_{\infty}<h$. We further restrict the choice so that $\left\|x_{h}-y_{h}\right\|_{2} \leqslant\|x-y\|_{2}$. Select $i, j \in\{1,2, \ldots, n\}$, and set $w=h z$, where $z=e_{i}$ or $z=e_{i}+e_{j}$. We have, denoting $z /\|z\|_{2}$ by $\hat{z}$, that

$$
\begin{aligned}
& \frac{\left|D_{\hat{z} \hat{z}} u_{\mathrm{e}}(x)-D_{\hat{z} \hat{z}} u_{\mathrm{e}}(y)\right|}{\|x-y\|_{2}^{\alpha}} \\
& \quad \leqslant \frac{\left|D_{\hat{z} \hat{z}} u_{\mathrm{e}}(x)-D_{\hat{z} \hat{z}} u_{\mathrm{e}}\left(x_{h}\right)\right|}{\|x-y\|_{2}^{\alpha}}+\frac{\left|D_{\hat{z} \hat{z}} u_{\mathrm{e}}\left(x_{h}\right)-\delta_{w}^{2} u\left(x_{h}\right)\right|}{\|x-y\|_{2}^{\alpha}}+\frac{\left|\delta_{w}^{2} u\left(x_{h}\right)-\delta_{w}^{2} u\left(y_{h}\right)\right|}{\|x-y\|_{2}^{\alpha}} \\
& \quad+\frac{\left|\delta_{w}^{2} u(y)-D_{\hat{z} \hat{z}} u_{\mathrm{e}}\left(y_{h}\right)\right|}{\|x-y\|_{2}^{\alpha}}+\frac{\left|D_{\hat{z} \hat{z}} u_{\mathrm{e}}\left(y_{h}\right)-D_{\hat{z} \hat{z}} u_{\mathrm{e}}(y)\right|}{\|x-y\|_{2}^{\alpha}}
\end{aligned}
$$

If $x_{h} \neq x$, then the first summand on the right-hand side satisfies

$$
\frac{\left|D_{\hat{z} \hat{z}} u_{\mathrm{e}}(x)-D_{\hat{z} \hat{z}} u_{\mathrm{e}}\left(x_{h}\right)\right|}{\|x-y\|_{2}^{\alpha}} \leqslant(\sqrt{n})^{\alpha} \frac{\left|D_{\hat{z} \hat{z}} u_{\mathrm{e}}(x)-D_{\hat{z} \hat{z}} u_{\mathrm{e}}\left(x_{h}\right)\right|}{\left\|x-x_{h}\right\|_{2}^{\alpha}} \leqslant 2 C n_{1}[u]_{2, \alpha ; \Omega_{h}},
$$

by case (i), using the triangle inequality and the fact that

$$
D_{\hat{z} \hat{z}} u_{\mathrm{e}} \equiv\left(2 D_{i j} u_{\mathrm{e}}+D_{i i} u_{\mathrm{e}}+D_{j j} u_{\mathrm{e}}\right) /\|z\|_{2}^{2}
$$

when $z=e_{i}+e_{j}$. The last summand on the right-hand side of (3.1) is dealt with similarly. The second summand on the right-hand side of (3.1) satisfies

$$
\frac{\left|D_{\hat{z} \hat{z}} u_{\mathrm{e}}\left(x_{h}\right)-\delta_{w}^{2} u\left(x_{h}\right)\right|}{\|x-y\|_{2}^{\alpha}}=\frac{\left|D_{\hat{z} \hat{z}} u_{\mathrm{e}}\left(x_{h}\right)-D_{\hat{z} \hat{z}} u_{\mathrm{e}}\left(x^{\prime}\right)\right|}{\|x-y\|_{2}^{\alpha}},
$$

by the mean value theorem where $x^{\prime}=x_{h}+t z, t \in(-1,1)$, and, hence,

$$
\left\|x_{h}-x^{\prime}\right\|_{2}<\sqrt{2} h<\sqrt{2}\|x-y\|_{\infty} \leqslant \sqrt{2}\|x-y\|_{2} .
$$

Thus, if $x^{\prime} \neq x_{h}$, the summand in question satisfies

$$
\frac{\left|D_{\hat{z} \hat{z}} u_{\mathrm{e}}\left(x_{h}\right)-\delta_{w}^{2} u\left(x_{h}\right)\right|}{\|x-y\|_{2}^{\alpha}} \leqslant \sqrt{2} \frac{\left|D_{\hat{z} \hat{z}} u_{\mathrm{e}}\left(x_{h}\right)-D_{\hat{z} \hat{z}} u_{\mathrm{e}}\left(x^{\prime}\right)\right|}{\left\|x_{h}-x^{\prime}\right\|_{2}^{\alpha}} \leqslant 2 \sqrt{2} C_{1}[u]_{2, \alpha ; \Omega_{h}},
$$


again by case (i) as above. The fourth summand on the right-hand side of (3.1) is dealt with in the same way. Finally, since $\left\|x_{h}-y_{h}\right\|_{2} \leqslant\|x-y\|_{2}$, if $x_{h} \neq y_{h}$, the third summand on the right-hand side of (3.1) clearly satisfies the same inequality. Ultimately we have

$$
\frac{\left|D_{\hat{z} \hat{z}} u_{\mathrm{e}}(x)-D_{\hat{z} \hat{z}} u_{\mathrm{e}}(y)\right|}{\|x-y\|_{2}^{\alpha}} \leqslant C_{1}[u]_{2, \alpha ; \Omega_{h}},
$$

which implies the same bound for $\left|D_{i j} u_{\mathrm{e}}(x)-D_{i j} u_{\mathrm{e}}(y)\right| /\|x-y\|_{2}^{\alpha}, i \neq j$, using the fact that

$$
D_{i j} u_{\mathrm{e}} \equiv \frac{1}{2}\left(D_{z z} u_{\mathrm{e}}-D_{i i} u_{\mathrm{e}}-D_{j j} u_{\mathrm{e}}\right)=\frac{1}{2}\left(\|z\|_{2}^{2} D_{\hat{z} \hat{z}} u_{\mathrm{e}}-D_{i i} u_{\mathrm{e}}-D_{j j} u_{\mathrm{e}}\right),
$$

when $z=e_{i}+e_{j}, i \neq j, i, j \in\{1,2, \ldots, n\}$. Taking the supremum over distinct $x, y \in \Omega$, $\|\beta\|_{1}=2$, we have the desired inequality.

Lemma 3.5. Let $u: \mathbb{Z}_{h}^{n} \rightarrow \mathbb{R}$ be a real-valued mesh function and suppose that $0<$ $\alpha<1$. There is a positive constant $C=C(n)$ such that

$$
C^{-1}\langle u\rangle_{\mathbb{R}^{n}}^{(2+\alpha)} \leqslant \underline{\mu}_{2+\alpha}(u) \leqslant C\langle u\rangle_{\mathbb{R}^{n}}^{(2+\alpha)}
$$

Proof. Certainly, since

$$
\langle u\rangle_{\mathbb{R}^{n}}^{(2+\alpha)}=\left\langle u_{\mathrm{e}}\right\rangle_{\mathbb{R}^{n}}^{(2+\alpha)}=\sup _{\|\beta\|_{1}=2}\left\langle D^{\beta} u_{\mathrm{e}}\right\rangle_{\mathbb{R}^{n}}^{(\alpha)}
$$

and $\left[u_{\mathrm{e}}\right]_{2, \alpha ; \mathbb{R}^{n}}=\sup _{\|\beta\|_{1}=2}\left[D^{\beta} u_{\mathrm{e}}\right]_{0, \alpha ; \mathbb{R}^{n}}$, it follows by the equivalence of semi-norms in (2.1) (see comment after (2.1)) that there exists a constant $C=C(n)$ such that

$$
\langle u\rangle_{\mathbb{R}^{n}}^{(2+\alpha)} \leqslant C\left[u_{\mathrm{e}}\right]_{2, \alpha ; \mathbb{R}^{n}} .
$$

Then by Theorem 3.4

$$
\langle u\rangle_{\mathbb{R}^{n}}^{(2+\alpha)} \leqslant C(n) \sup _{x, y \in \mathbb{Z}_{h}^{n}, x \neq y, z \in Y_{1}} \frac{\left|\delta_{z}^{2} u_{\mathrm{e}}(x)-\delta_{z}^{2} u_{\mathrm{e}}(y)\right|}{\|x-y\|_{2}^{\alpha}} .
$$

By the mean value theorem, for fixed $x, y, z$, there exists $t \in\{x+s z: s \in(-1,1)\}$ such that $\delta_{z}^{2} u_{\mathrm{e}}(x)=D_{\hat{z} \hat{z}} u_{\mathrm{e}}(t)$, where $\hat{z}=z /\|z\|_{2}$, and there exists $r \in\{y+s z: s \in(-1,1)\}$ such that $\delta_{z}^{2} u_{\mathrm{e}}(y)=D_{\hat{z} \hat{z}} u_{\mathrm{e}}(r)$. If $\|t-r\|_{2} \leqslant\|x-y\|_{2}$ and $t \neq r$ (if $t=r$, then $\delta_{z}^{2} u_{\mathrm{e}}(x)=$ $\left.\delta_{z}^{2} u_{\mathrm{e}}(y)\right)$, then, with $\rho=\|x-y\|_{2} \geqslant h$,

$$
\frac{\left|\delta_{z}^{2} u_{\mathrm{e}}(x)-\delta_{z}^{2} u_{\mathrm{e}}(y)\right|}{\|x-y\|_{2}^{\alpha}} \leqslant \frac{\operatorname{osc}_{K_{\rho}(t)} D_{\hat{z} \hat{z}} u_{\mathrm{e}}}{\rho^{\alpha}} \leqslant n \sup _{\|\beta\|_{1}=2} \frac{\operatorname{osc}_{K_{\rho}(t)} D^{\beta} u_{\mathrm{e}}}{\rho^{\alpha}},
$$

and if $\rho=\|t-r\|_{2}>\|x-y\|_{2}$, in which case

$$
\frac{1}{\|x-y\|_{2}} \leqslant \frac{1+2 \sqrt{n}}{\|t-r\|_{2}}
$$


we have

$$
\begin{aligned}
\frac{\left|\delta_{z}^{2} u_{\mathrm{e}}(x)-\delta_{z}^{2} u_{\mathrm{e}}(y)\right|}{\|x-y\|_{2}^{\alpha}} & \leqslant(1+2 \sqrt{n})^{\alpha} \frac{\operatorname{osc}_{K_{\rho}(t)} D_{\hat{z} \hat{z}} u_{\mathrm{e}}}{\rho^{\alpha}} \\
& \leqslant n(1+2 \sqrt{n}) \sup _{\|\beta\|_{1}=2} \frac{\operatorname{osc}_{K_{\rho}(t)} D^{\beta} u_{\mathrm{e}}}{\rho^{\alpha}} .
\end{aligned}
$$

This together with (3.4) implies that there exists positive $C=C(n)$ such that for all $x, y \in \mathbb{Z}_{h}^{n}, z \in Y_{1}$,

$$
\begin{aligned}
\frac{\left|\delta_{z}^{2} u_{\mathrm{e}}(x)-\delta_{z}^{2} u_{\mathrm{e}}(y)\right|}{\|x-y\|_{2}^{\alpha}} & \leqslant C \sup _{\substack{\|\beta\|_{1}=2, t \in \mathbb{R}^{n} \\
\rho \geqslant h}} \frac{\operatorname{osc}_{K_{\rho}(t)} D^{\beta} u_{\mathrm{e}}}{\rho^{\alpha}} \\
& =C \underline{\mu}_{2+\alpha}(u) .
\end{aligned}
$$

Taking the supremum over all such $x, y$ and $z$ we obtain from (3.3) the left-hand inequality in (3.2). The reverse inequality with $C=1$ is obvious.

Lemma 3.6. Let $u: \mathbb{Z}_{h}^{n} \rightarrow \mathbb{R}$ be a real-valued mesh function and suppose that $0<$ $\alpha<1$. There is a positive constant $C=C(n, \alpha)$ such that

$$
C^{-1}\langle u\rangle_{\mathbb{R}^{n}}^{(2+\alpha)} \leqslant \underline{\mu}_{2+\alpha}^{*}(u) \leqslant C\langle u\rangle_{\mathbb{R}^{n}}^{(2+\alpha)} .
$$

Proof. In the following, the first inequality is obvious, and the second follows from (2.3) with $v \equiv u_{\mathrm{e}}$ :

$$
\underline{\mu}_{2+\alpha}^{*}(u) \leqslant \mu_{2+\alpha}^{*}(u) \leqslant C\langle u\rangle_{\mathbb{R}^{n}}^{(2+\alpha)},
$$

giving the right-hand inequality in the statement of the lemma.

For the opposite inequality, we establish $\underline{\mu}_{2+\alpha}(u) \leqslant C \underline{\mu}_{2+\alpha}^{*}(u)$ by following Safonov's $\left[\mathbf{1 2}\right.$, Theorem $\left.2.1^{\prime}\right]$ proof of the equivalence

$$
\langle v\rangle_{\mathbb{R}^{n}}^{(m+\alpha)} \sim \mu_{m+\alpha}^{*}(v)
$$

for general $v \in C^{m, \alpha}\left(\mathbb{R}^{n}\right)$ satisfying $\langle v\rangle_{\mathbb{R}^{n}}^{(m+\alpha)}<\infty$. We use Lemmas 3.2 and 3.5 to do this. A further application of Lemma 3.5 then gives us the desired inequality.

Specifically, by Lemma 3.2 and then Lemma 3.5 we have, for $\rho \geqslant h$ and $x \in \mathbb{R}^{n}$,

$$
\begin{aligned}
\rho^{-\alpha} \omega_{2}\left(u_{\mathrm{e}}, K_{\rho}(x)\right) & \leqslant \varepsilon\langle u\rangle_{\mathbb{R}^{n}}^{(2+\alpha)}+C(\varepsilon, n, \alpha) \underline{\mu}_{2+\alpha}^{*}(u) \\
& \leqslant \varepsilon C^{\prime}(n) \underline{\mu}_{2+\alpha}(u)+C(\varepsilon, n, \alpha) \underline{\mu}_{2+\alpha}^{*}(u),
\end{aligned}
$$

for any $\varepsilon>0$. It follows that

$$
\underline{\mu}_{2+\alpha}(u) \leqslant C^{\prime}(n) \varepsilon \underline{\mu}_{2+\alpha}(u)+C(n, \varepsilon, \alpha) \underline{\mu}_{2+\alpha}^{*}(u) .
$$

Setting $\varepsilon=1 /\left(2 C^{\prime}\right)$ and applying Lemma 3.5 to the left-hand side we obtain $C=$ $C(n, \alpha)>0$ such that

$$
\langle u\rangle_{\mathbb{R}^{n}}^{(2+\alpha)} \leqslant C \underline{\mu}_{2+\alpha}^{*}(u),
$$

the left-hand inequality in the statement of the lemma. 


\section{4. $C^{2, \alpha}$ estimate in/on $\mathbb{Z}_{h}^{n}$ : explicit $x$-dependence}

Theorem 4.1. Let $u: \mathbb{Z}_{h}^{n} \rightarrow \mathbb{R}$ be a mesh function solution of

$$
F_{h}[u](x) \equiv \mathcal{F}_{h}\left(x, \delta^{2} u(x)\right)=0, \quad \forall x \in \mathbb{Z}_{h}^{n},
$$

where $\mathcal{F}_{h}(x, \cdot) \in C^{1}\left(\mathbb{R}^{Y^{\prime}}\right)$ for each $x \in \mathbb{Z}_{h}^{n},\left\{h e_{i}\right\}_{i=1}^{n} \subset Y^{\prime} \subset Y_{N}$, and $\delta^{2} u(x)=\left\{\delta_{y}^{2} u(x) \mid\right.$ $\left.y \in Y^{\prime}\right\}$. Suppose that there exist $\lambda, \Lambda>0$ such that, for each $(x, s) \in \mathbb{Z}_{h}^{n} \times \mathbb{R}^{Y^{\prime}}$,

$$
\lambda \leqslant \frac{\partial \mathcal{F}_{h}(x, s)}{\partial s_{y}} \leqslant \Lambda, \quad \forall y \in Y^{\prime} .
$$

Assume that for each $x \in \mathbb{Z}_{h}^{n}, \mathcal{F}_{h}(x, s)$ is concave in $s$, and that $\mathcal{F}_{h}$ is symmetric with respect to $s_{ \pm y}$ for all $y \in Y^{\prime}$. Assume, further, that for some $\gamma \in(0, \alpha)$, where $\alpha=$ $\alpha(n, N, \lambda, \Lambda) \in(0,1)$ is the constant of [6, Theorem 3.2], there exist $k_{1}, k_{2}>0$ such that, for all $\left\{s_{y}\right\} \in \mathbb{R}^{Y^{\prime}}$,

$$
\left[\mathcal{F}_{h}\left(\cdot,\left\{s_{y}\right\}\right)\right]_{0, \gamma ; \mathbb{Z}_{h}^{n}} \leqslant k_{1} \sum_{y \in Y^{\prime}}\left|s_{y}\right|+k_{2}
$$

where

$$
\left[\mathcal{F}_{h}\left(\cdot,\left\{s_{y}\right\}\right)\right]_{0, \gamma ; \mathbb{Z}_{h}^{n}}=\sup _{x, z \in \mathbb{Z}_{h}^{n}, x \neq z} \frac{\left|\mathcal{F}_{h}\left(x,\left\{s_{y}\right\}\right)-\mathcal{F}_{h}\left(z,\left\{s_{y}\right\}\right)\right|}{\|x-z\|_{2}^{\gamma}} ;
$$

then, there exists a positive constant $C=C\left(n, N, \lambda, \Lambda, k_{1}, \gamma\right)$ such that

$$
{ }_{N}[u]_{2, \gamma ; \mathbb{Z}_{h}^{n}} \leqslant C\left({ }_{N}[u]_{2 ; \mathbb{Z}_{h}^{n}}+k_{2}\right) .
$$

If the semi-norms above are finite and $|u|_{0 ; \mathbb{Z}_{h}^{n}}$ is finite and $0<h \leqslant h_{0}$, then

$$
{ }_{N}[u]_{2, \gamma ; \mathbb{Z}_{h}^{n}} \leqslant C\left(|u|_{0 ; \mathbb{Z}_{h}^{n}}+k_{2}\right),
$$

where $C$ depends in addition upon $h_{0}$.

Proof of Theorem 4.1. We first prove (4.3) in the case when $\mathcal{F}_{h}$ is diagonal, that is, $N=1$ and $\mathcal{F}_{h}$ depends only upon difference quotients $\left\{\delta_{ \pm h e_{1}}^{2} u, \ldots, \delta_{ \pm h e_{n}}^{2} u\right\}$ (and $x$, of course). Our proof of this case is a discretization of Safonov's derivation in the corresponding situation for partial differential equations $[\mathbf{1 1}, \mathbf{1 2}]$.

Let $u: \mathbb{Z}_{h}^{n} \rightarrow \mathbb{R}$ be a solution of (4.1), where $h>0$ is fixed. Let $u_{\mathrm{e}} \in C^{\infty}\left(\mathbb{R}^{n}\right)$ be the Kunkle extension of $u$ to $\mathbb{R}^{n}$, as provided in Lemma 1.1. Fix $\bar{x} \in \mathbb{R}^{n}, \rho \geqslant h$ and $\varepsilon \in\left(0, \frac{1}{6}\right]$. Let $r=\rho / \varepsilon$, in which case $r \geqslant 6 h$.

Choose $\boldsymbol{x} \in \mathbb{Z}_{h}^{n}$ so that $\|\boldsymbol{x}-\bar{x}\|_{\infty} \leqslant h$. Let $\boldsymbol{r}$ be the smallest even multiple of $h$ larger than or equal to $r$. We have, of course, that $\boldsymbol{r} / 2=m h$ for some $m \in \mathbb{N}$, and, since $r \geqslant 6 h$, we have $\boldsymbol{r} / 2 \geqslant 3 h$. Note also that $\rho / \boldsymbol{r} \leqslant \rho / r=\varepsilon$, and $\boldsymbol{r}<r+2 h \leqslant r+2 r / 6=4 r / 3$, and hence

$$
\frac{3}{4} \varepsilon \leqslant(\rho / \boldsymbol{r}) \leqslant \varepsilon
$$


It then follows that

$$
K_{\rho}(\bar{x}) \subset K_{r / 2}(\boldsymbol{x}) \subset K_{r / 2}(\boldsymbol{x})
$$

Define

$$
\varphi=u_{\mathrm{e}}-T_{\boldsymbol{x}, 2} u_{\mathrm{e}},
$$

where $T_{\boldsymbol{x}, 2} u_{\mathrm{e}}$ is the Taylor polynomial of $u_{\mathrm{e}}$ about $\boldsymbol{x}$ of total degree at most 2 (see (2.2)). Let the mesh function $v: K_{r}(\boldsymbol{x})_{h} \rightarrow \mathbb{R}$ be the solution of the (frozen) Dirichlet problem

$$
\left.\begin{array}{rlrl}
F_{0 h}[v](x) \equiv \mathcal{F}_{h 0}\left(\delta^{2} v(x)\right)=\mathcal{F}_{h}\left(\boldsymbol{x}, \delta^{2} u(\boldsymbol{x})+\delta^{2} v(x)\right) & =0, & & \forall x \in K_{\boldsymbol{r}}(\boldsymbol{x})_{h}^{i}, \\
v & =\varphi, & & \forall x \in K_{\boldsymbol{r}}(\boldsymbol{x})_{h}^{b},
\end{array}\right\}
$$

where

$$
\Omega_{h}^{i}=\left\{x \in \Omega_{h} \mid x+y \in \Omega_{h}, \forall y \in Y_{1}\right\}, \quad \Omega_{h}^{b}=\Omega_{h} \backslash \Omega_{h}^{i} .
$$

The existence of $v$ follows from [6, Theorem 1.1] (see [10, Theorem 3.6]), noting that in the case where $\mathcal{F}_{h_{0}}$ is diagonal, the interior of $K_{\boldsymbol{r}}(\boldsymbol{x})_{h}$ relative to $\mathcal{F}_{h_{0}}$ (see [6]) coincides with $K_{\boldsymbol{r}}(\boldsymbol{x})_{h}^{i}$, and hence also the boundary of $K_{\boldsymbol{r}}(\boldsymbol{x})_{h}$ relative to $\mathcal{F}_{h_{0}}$ coincides with $K_{\boldsymbol{r}}(\boldsymbol{x})_{h}^{b}$. Let $v_{\mathrm{e}}$ be the Kunkle extension of $v$ to the closed cube with mesh point corners $K_{\boldsymbol{r}}(\boldsymbol{x})$, as provided in Lemma 1.1. Let $\gamma \in(0, \alpha)$ be such that $(4.2)$ is satisfied. Then, with a view to estimating $\underline{\mu}_{2+\gamma}^{*}(u)$, we have

$$
\begin{aligned}
\rho^{-2-\gamma} E_{2}\left[u_{\mathrm{e}} ; K_{\rho}(\bar{x})\right] & =\rho^{-2-\gamma} E_{2}\left[\varphi ; K_{\rho}(\bar{x})\right] \\
& \leqslant \rho^{-2-\gamma} E_{2}\left[v_{\mathrm{e}} ; K_{\rho}(\bar{x})\right]+\rho^{-2-\gamma} E_{2}\left[\varphi-v_{\mathrm{e}} ; K_{\rho}(\bar{x})\right] .
\end{aligned}
$$

Dealing with the first summand on the right-hand side we have by Lemma 3.3, (4.6), equivalence of semi-norms in (2.1), and Theorem 3.4, in turn, that

$$
\begin{aligned}
\rho^{-2-\gamma} E_{2}\left[v_{\mathrm{e}} ; K_{\rho}(\bar{x})\right] & \leqslant C(n) \rho^{\alpha-\gamma}\left\langle v_{\mathrm{e}}\right\rangle_{K_{\rho}(\bar{x})}^{(2+\alpha)} \leqslant C \rho^{\alpha-\gamma}\left\langle v_{\mathrm{e}}\right\rangle_{K_{r / 2}(\boldsymbol{x})}^{(2+\alpha)} \\
& \leqslant C \rho^{\alpha-\gamma}\left[v_{\mathrm{e}}\right]_{2, \alpha ; K_{r / 2}(\boldsymbol{x})} \leqslant C(n) \rho^{\alpha-\gamma}{ }_{1}[v]_{2, \alpha ; K_{r / 2}(\boldsymbol{x})_{h}} .
\end{aligned}
$$

Applying Theorem 3.2 of $[\mathbf{6}]$ to $F_{0 h}$, which satisfies $F_{0 h}[0]=0$, it follows from the above that

$$
\rho^{-2-\gamma} E_{2}\left[v_{\mathrm{e}} ; K_{\rho}(\bar{x})\right] \leqslant C(n, \lambda, \Lambda) \rho^{\alpha-\gamma} \boldsymbol{r}^{-2-\alpha} \max _{K_{\boldsymbol{r}}(\boldsymbol{x})_{h}^{b}}|\varphi| .
$$

Then the definition of $\varphi$, Lemma 3.3 and (4.5) give the following

$$
\rho^{-2-\gamma} E_{2}\left[v_{\mathrm{e}} ; K_{\rho}(\bar{x})\right] \leqslant C \rho^{\alpha-\gamma} \boldsymbol{r}^{-2-\alpha} \boldsymbol{r}^{2+\gamma}\left\langle u_{\mathrm{e}}\right\rangle_{K_{r}(\boldsymbol{x})}^{(2+\gamma)} \leqslant C(n, \lambda, \Lambda) \varepsilon^{\alpha-\gamma}\left\langle u_{\mathrm{e}}\right\rangle_{K_{r}(\boldsymbol{x})}^{(2+\gamma)} .
$$

Now consider the second summand on the right-hand side of (4.8). By definition of $E_{2}$ and $\varphi$, by (4.6) and by the final paragraph of Lemma 1.1, we have

$$
\begin{aligned}
\rho^{-2-\gamma} E_{2}\left[\varphi-v_{\mathrm{e}} ; K_{\rho}(\bar{x})\right] & \leqslant \rho^{-2-\gamma} \sup _{K_{r}(\boldsymbol{x})}\left|u_{\mathrm{e}}-T_{\boldsymbol{x}, 2} u_{\mathrm{e}}-v_{\mathrm{e}}\right| \\
& \leqslant C(n) \rho^{-2-\gamma} \max _{K_{r}(\boldsymbol{x})_{h}}|\varphi-v| .
\end{aligned}
$$


Now we estimate $\varphi-v$ on $K_{\boldsymbol{r}}(\boldsymbol{x})_{h}$. We have by linearization (see the proof of Theorem 3.2 in $[\mathbf{6}]$ and $[\mathbf{1 0}, \S 3])$

$$
F_{0 h}[\varphi]=F_{0 h}[\varphi]-F_{0 h}[v]=L_{0 h}(\varphi-v), \quad \forall x \in K_{\boldsymbol{r}}(\boldsymbol{x})_{h}^{i},
$$

where $L_{0 h}=\sum_{i=1}^{n} a\left(x, h e_{i}\right) \delta_{h e_{i}}^{2}$ and

$$
a(x, y)=\int_{0}^{1} \frac{\partial \mathcal{F}_{h 0}\left(\delta^{2} w_{t}\right)}{\partial s_{y}} \mathrm{~d} t, \quad w_{t}=t \varphi(x)+(1-t) v(x), \quad 0 \leqslant t \leqslant 1,
$$

and of course, by the assumptions on $F_{h}, a(x, y) \geqslant \lambda>0$. Letting

$$
A=\boldsymbol{r}^{-\gamma} \max _{K_{\boldsymbol{r}}(\boldsymbol{x})_{h}^{i}}\left|F_{0 h}[\varphi]\right|
$$

we have $\left|L_{0 h}(\varphi-v)\right| \leqslant A \boldsymbol{r}^{\gamma}$ in $K_{\boldsymbol{r}}(\boldsymbol{x})_{h}^{i}$, and, by definition, $\varphi-v=0$ for all $x \in K_{\boldsymbol{r}}(\boldsymbol{x})_{h}^{b}$. We compare $\varphi-v$ with the function

$$
w(x)=\frac{A \boldsymbol{r}^{\gamma}}{2 n \lambda}\left((\sqrt{n} \boldsymbol{r})^{2}-\|x-\boldsymbol{x}\|_{2}^{2}\right) .
$$

Note that $\|x-\boldsymbol{x}\|_{2}^{2} \leqslant n \boldsymbol{r}^{2}$ for all $x \in K_{\boldsymbol{r}}(\boldsymbol{x})_{h}^{b}$, so that $w(x) \geqslant 0$ for all $x \in K_{\boldsymbol{r}}(\boldsymbol{x})_{h}^{b}$. By elementary calculation we find that

$$
\delta_{y}^{2} w(x)=\frac{-A \boldsymbol{r}^{\gamma}}{n \lambda},
$$

for any $y \neq 0$. Therefore,

$$
\begin{aligned}
L_{0 h} w(x)=\sum_{i=1}^{n} a\left(x, h e_{i}\right) \delta_{h e_{i}}^{2} w(x) & =\frac{-A \boldsymbol{r}^{\gamma}}{n \lambda} \sum_{i=1}^{n} a\left(x, h e_{i}\right) \\
& \leqslant-A \boldsymbol{r}^{\gamma} .
\end{aligned}
$$

Hence,

$$
L_{0 h}( \pm(\varphi-v)-w)= \pm L_{0 h}(\varphi-v)-L_{0 h} w \geqslant-A \boldsymbol{r}^{\gamma}+A \boldsymbol{r}^{\gamma}=0 .
$$

Applying the discrete maximum principle, [9, Theorem 2.1], and denoting $\max \{f, 0\}$ by $f^{+}$, we obtain

$$
\max _{K_{r}(\boldsymbol{x})_{h}^{i}}(|\varphi-v|-w) \leqslant \max _{K_{r}(\boldsymbol{x})_{h}^{b}}(|\varphi-v|-w)^{+},
$$

which must be zero since $\varphi-v=0$ for all $x \in K_{\boldsymbol{r}}(\boldsymbol{x})_{h}^{b}$ and $w \geqslant 0$ for all $x \in K_{\boldsymbol{r}}(\boldsymbol{x})_{h}^{b}$. It follows that

$$
\max _{K_{r}(\boldsymbol{x})_{h}}|\varphi-v| \leqslant \max _{K_{r}(\boldsymbol{x})_{h}} w \leqslant \frac{A \boldsymbol{r}^{\gamma}}{2 n \lambda} n \boldsymbol{r}^{2}=\frac{1}{2 \lambda} A \boldsymbol{r}^{2+\gamma} .
$$

Now let us recall the concluding paragraph of Lemma 1.1, from which we see that for each $i=1, \ldots, n$, the function $\varphi$ as defined here satisfies

$$
\delta_{ \pm h e_{i}}^{2} \varphi(x)=\delta_{ \pm h e_{i}}^{2} u_{\mathrm{e}}(x)-\delta_{ \pm h e_{i}}^{2} T_{\boldsymbol{x}, 2} u_{\mathrm{e}}(x)=\delta_{ \pm h e_{i}}^{2} u_{\mathrm{e}}(x)-\delta_{ \pm h e_{i}}^{2} u_{\mathrm{e}}(\boldsymbol{x}), \quad \forall x \in \mathbb{Z}_{h}^{n}
$$


that is,

$$
\delta^{2} \varphi(x)=\delta^{2} u_{\mathrm{e}}(x)-\delta^{2} u_{\mathrm{e}}(\boldsymbol{x}), \quad \forall x \in \mathbb{Z}_{h}^{n},
$$

since $F_{h}$ is diagonal. From (4.7) it follows that for all $x \in K_{\boldsymbol{r}}(\boldsymbol{x})_{h}^{i}$,

$$
F_{0 h}[\varphi](x)=\mathcal{F}_{h}\left(\boldsymbol{x}, \delta^{2} u(\boldsymbol{x})+\delta^{2} u(x)-\delta^{2} u(\boldsymbol{x})\right)=\mathcal{F}_{h}\left(\boldsymbol{x}, \delta^{2} u(x)\right),
$$

and, hence, since $F_{h}[u] \equiv 0$,

$$
\left|F_{0 h}[\varphi](x)\right|=\left|F_{0 h}[\varphi](x)-F_{h}[u](x)\right|=\left|\mathcal{F}_{h}\left(\boldsymbol{x}, \delta^{2} u(x)\right)-\mathcal{F}_{h}\left(x, \delta^{2} u(x)\right)\right| .
$$

Then, with $\boldsymbol{r}>h$, we have, by (4.2), that

$$
\begin{aligned}
A=\boldsymbol{r}^{-\gamma} \max _{K_{\boldsymbol{r}}(\boldsymbol{x})_{h}^{i}}\left|F_{0 h}[\varphi]\right| & \leqslant \max _{K_{\boldsymbol{r}}(\boldsymbol{x})_{h}^{i} \backslash\{\boldsymbol{x}\}} \frac{\left|\mathcal{F}_{h}\left(\boldsymbol{x}, \delta^{2} u(x)\right)-\mathcal{F}_{h}\left(x, \delta^{2} u(x)\right)\right|}{\|x-\boldsymbol{x}\|_{\infty}^{\gamma}} \\
& \leqslant(\sqrt{n})^{\gamma} \max _{K_{\boldsymbol{r}}(\boldsymbol{x})_{h}^{i} \backslash\{\boldsymbol{x}\}} \frac{\left|\mathcal{F}_{h}\left(\boldsymbol{x}, \delta^{2} u(x)\right)-\mathcal{F}_{h}\left(x, \delta^{2} u(x)\right)\right|}{\|x-\boldsymbol{x}\|_{2}^{\gamma}} \\
& \leqslant \sqrt{n} \max _{K_{r}(\boldsymbol{x})_{h}^{i}}\left(k_{1} \sum_{i=1}^{n}\left|\delta_{ \pm h e_{i}}^{2} u(x)\right|+k_{2}\right) .
\end{aligned}
$$

Note that the maximum above is over the interior, $K_{\boldsymbol{r}}(\boldsymbol{x})_{h}^{i}$, of $K_{\boldsymbol{r}}(\boldsymbol{x})_{h}$, so that for each $x$ in this interior, the support of $\delta_{h e_{i}}^{2} u(x)$ is contained in $K_{\boldsymbol{r}}(\boldsymbol{x})_{h}$. We may therefore conclude that $A \leqslant \sqrt{n}\left(2 n k_{1+}[u]_{2 ; K_{h}^{r}(\boldsymbol{x})}+k_{2}\right)$ (see $[\mathbf{6}, \S 1.1]$ for definition of semi-norm). Inserting this in (4.11) gives

$$
\max _{K_{r}(\boldsymbol{x})_{h}}|\varphi-v| \leqslant \frac{\sqrt{n}}{2 \lambda} \boldsymbol{r}^{2+\gamma}\left(2 n k_{1+}[u]_{2 ; K_{r}(\boldsymbol{x})_{h}}+k_{2}\right) .
$$

Returning, then, to (4.10), we deduce, using the above and (4.5), that

$$
\rho^{-2-\gamma} E_{2}\left[\varphi-v_{\mathrm{e}} ; K_{\rho}(\bar{x})\right] \leqslant C(n, \lambda) \varepsilon^{-2-\gamma}\left(2 n k_{1+}[u]_{2 ; K_{r}(\boldsymbol{x})_{h}}+k_{2}\right) .
$$

This with (4.9) allows us to rewrite (4.8):

$$
\begin{aligned}
\rho^{-2-\gamma} E_{2}\left[u_{\mathrm{e}} ; K_{\rho}(\bar{x})\right] & \leqslant C(n, \lambda, \Lambda) \varepsilon^{\alpha-\gamma}\left\langle u_{\mathrm{e}}\right\rangle_{K_{r}(\boldsymbol{x})}^{(2+\gamma)}+C^{\prime}(n, \lambda) \varepsilon^{-2-\gamma}\left(2 n k_{1+}[u]_{2 ; K_{r}(\boldsymbol{x})_{h}}+k_{2}\right) \\
& \leqslant C \varepsilon^{\alpha-\gamma}\left\langle u_{\mathrm{e}}\right\rangle_{\mathbb{R}^{n}}^{(2+\gamma)}+C^{\prime} \varepsilon^{-2-\gamma}\left(2 n k_{1}+[u]_{2 ; \mathbb{Z}_{h}^{n}}+k_{2}\right) .
\end{aligned}
$$

Now take the supremum on the left-hand side over all $\rho \geqslant h, \bar{x} \in \mathbb{R}^{n}$, to find that

$$
\underline{\mu}_{2+\gamma}^{*}(u) \leqslant C \varepsilon^{\alpha-\gamma}\left\langle u_{\mathrm{e}}\right\rangle_{\mathbb{R}^{n}}^{2+\gamma}+C^{\prime} \varepsilon^{-2-\gamma}\left(2 n k_{1+}[u]_{2 ; \mathbb{Z}_{h}^{n}}+k_{2}\right) .
$$

It then follows from this and Lemma 3.6 (and the fact that $\alpha=\alpha(n, \lambda, \Lambda)$ ) that there are positive constants $C_{1}=C_{1}(n, \lambda, \Lambda)$ and $C_{2}=C_{2}(n, \lambda, \Lambda)$ such that

$$
\left\langle u_{\mathrm{e}}\right\rangle_{\mathbb{R}^{n}}^{2+\gamma} \leqslant C_{1} \varepsilon^{\alpha-\gamma}\left\langle u_{\mathrm{e}}\right\rangle_{\mathbb{R}^{n}}^{2+\gamma}+C_{2} \varepsilon^{-2-\gamma}\left(2 n k_{1+}[u]_{2 ; \mathbb{Z}_{h}^{n}}+k_{2}\right) .
$$


Now choose $\varepsilon$ small enough so that $C_{1} \varepsilon^{\alpha-\gamma}<1$, and it follows that

$$
\left\langle u_{\mathrm{e}}\right\rangle_{\mathbb{R}^{n}}^{2+\gamma} \leqslant C\left(2 n k_{1+}[u]_{2 ; \mathbb{Z}_{h}^{n}}+k_{2}\right),
$$

$C$ depending only upon $n, \lambda, \Lambda$ and $\gamma$. Finally, we move to the mesh function $u$ on the left by the equivalence of semi-norms in (2.1) and in Theorem 3.4 for the extension $u_{\mathrm{e}}$, thus establishing

$$
{ }_{N}[u]_{2, \gamma ; \mathbb{Z}_{h}^{n}} \leqslant C\left(2 n k_{1+}[u]_{2 ; \mathbb{Z}_{h}^{n}}+k_{2}\right),
$$

for $\mathcal{F}_{h}$ explicitly dependent upon $x$ and upon centred second-order difference quotients in directions $\left\{ \pm h e_{1}, \ldots, \pm h e_{n}\right\}$ only.

If $\mathcal{F}_{h}$ is not diagonal, that is, if $\mathcal{F}_{h}$ depends upon centred second-order difference quotients other than $\delta_{ \pm h e_{i}}^{2} u$ for $i=1, \ldots, n$, then (4.12) is not true, in general.

We can rectify the foregoing proof for the more general equation where $\mathcal{F}_{h}$ does depend upon some $\delta_{y}^{2} u$ for $y \in Y_{N} \backslash\left\{ \pm h e_{1}, \ldots, \pm h e_{n}\right\}$. Specifically, our approach is to transform the equation $\mathcal{F}_{h}\left(x, \delta^{2} u(x)\right)=0$ to a higher dimension so that the directions $y \in Y^{\prime}$ are mapped to orthogonal directions in the higher dimension and, hence, the discrete Hessian becomes discretely local (that is, (4.12) is true); the equation becomes diagonal.

In $Y^{\prime} \subset Y_{N}=\left\{z \in \mathbb{Z}_{h}^{n} \mid 0<\|z\|_{\infty} \leqslant N h\right\}$ there are at most $(2 N+1)^{n}-1$ nonzero directions, where, if $y \in Y^{\prime}$, then $-y \in Y^{\prime}$ (recall the symmetry assumed of $\mathcal{F}_{h}$ with respect to $s_{ \pm y}$ ). We choose half of these directions so that if $y$ is chosen, then $-y$ is excluded. Defining $M \leqslant\left[(2 N+1)^{n}-1\right] / 2$ to be half the number of directions in $Y^{\prime}$, we transform our difference equation in $\mathbb{Z}_{h}^{n}$ dependent upon $x \in \mathbb{Z}_{h}^{n}$ and the centred second-order difference quotients in directions $y \in Y^{\prime}$ to a difference equation in $\mathbb{Z}_{h}^{M}$ dependent upon $x \in \mathbb{Z}_{h}^{M}$ and the centred second-order difference quotients in the orthogonal directions and their negatives. We map each direction $y \in Y^{\prime}$ to an orthogonal direction $\pm h e_{i} \in \mathbb{Z}_{h}^{M}$. Specifically, choose all $y \in Y^{\prime}$ such that the first nonzero component (with respect to the standard basis) of $y$ is positive, and enumerate these as $\left\{y^{1}, y^{2}, \ldots, y^{M}\right\}$. We now associate $y^{i}$ with $h e_{i} \in \mathbb{Z}_{h}^{M}$ for each $i=1, \ldots, M$. If $u: \mathbb{Z}_{h}^{n} \rightarrow \mathbb{R}$ is an $n$-dimensional mesh function, then define the $M$-dimensional mesh function $u_{M}: \mathbb{Z}_{h}^{M} \rightarrow \mathbb{R}$ as

$$
u_{M}\left(x_{1}, \ldots, x_{M}\right)=u\left(\sum_{i=1}^{M} x_{i} y^{i} / h\right),
$$

for each $x=\left(x_{1}, \ldots, x_{M}\right) \in \mathbb{Z}_{h}^{M}$. Observe that if on the left-hand side we increase $x_{j}$ by $h$, on the right-hand side the argument is incremented by $y^{j}$, and we would expect that orthogonal difference on the left-hand side would correspond to differences in the directions $y^{j} \in Y^{\prime}$ on the right-hand side, modulo a constant depending upon $N$ and $n$. Making this explicit, we have

$$
\delta_{ \pm h e_{j}}^{2} u_{M}(x)=\frac{\left\|y^{j}\right\|_{2}^{2}}{h^{2}} \delta_{ \pm y^{j}}^{2} u\left(\sum_{i=1}^{M} x_{i} y^{i} / h\right) .
$$

With $y^{j} \in Y^{\prime}$ we have $h \leqslant\left\|y^{j}\right\|_{2} \leqslant N h \sqrt{n}$, and hence

$$
1 \leqslant \frac{\left\|y^{j}\right\|_{2}^{2}}{h^{2}} \leqslant n N^{2}
$$


Notice that (4.14) and (4.15) imply that

$$
+\left[u_{M}\right]_{2 ; \mathbb{Z}_{h}^{M}} \leqslant n N^{2}{ }_{N}[u]_{2 ; \mathbb{Z}_{h}^{n}} .
$$

Also, by our choice of the $y^{i}$ there exist $i_{1}, i_{2}, \ldots, i_{n} \in\{1, \ldots, M\}$ such that $y^{i_{j}}=h e_{j}$, $j=1,2, \ldots, n$. Suppose, without loss of generality, that $i_{j}=j$; that is, suppose that $y^{1}=h e_{1}, y^{2}=h e_{2}, \ldots, y^{n}=h e_{n}$. Then

$$
u\left(x_{1}, x_{2}, \ldots, x_{n}\right)=u_{M}\left(x_{1}, x_{2}, \ldots, x_{n}, 0,0, \ldots, 0\right),
$$

for all $x=\left(x_{1}, x_{2}, \ldots, x_{n}\right) \in \mathbb{Z}_{h}^{n}$. It follows that

$$
{ }_{N}[u]_{2, \gamma ; \mathbb{Z}_{h}^{n}} \leqslant N_{N}\left[u_{M}\right]_{2, \gamma ; \mathbb{Z}_{h}^{M}} .
$$

Now, with $\mathcal{F}_{h}$ as in the hypothesis of the theorem, arguments ordered as follows:

$$
\mathcal{F}_{h}(\cdot, s)=\mathcal{F}_{h}\left(\cdot, s_{y^{1}}, s_{y^{2}}, \ldots, s_{y^{M}}, s_{-y^{1}}, s_{-y^{2}}, \ldots, s_{-y^{M}}\right),
$$

define for $x \in \mathbb{Z}_{h}^{M},\left(s_{1}, s_{2}, \ldots, s_{M}, s_{-1}, s_{-2}, \ldots, s_{-M}\right) \in \mathbb{R}^{2 M}$,

$$
\mathcal{F}_{h M}\left(x,\left\{s_{ \pm i}\right\}_{i=1}^{M}\right)=\mathcal{F}_{h}\left(\sum_{i=1}^{M} x_{i} y^{i} / h,\left\{\frac{h^{2}}{\left\|y^{i}\right\|_{2}^{2}} s_{ \pm i}\right\}_{i=1}^{M}\right) .
$$

Then

$$
\frac{\partial \mathcal{F}_{h M}}{\partial s_{ \pm i}}=\frac{h^{2}}{\left\|y^{i}\right\|_{2}^{2}} \frac{\partial \mathcal{F}_{h}}{\partial s_{ \pm y^{i}}},
$$

and so (4.15) and the fact that $\lambda \leqslant \partial \mathcal{F}_{h} / \partial s_{ \pm y^{i}} \leqslant \Lambda$ imply that

$$
\frac{\lambda}{n N^{2}} \leqslant \frac{\partial \mathcal{F}_{h M}}{\partial s_{ \pm i}} \leqslant \Lambda
$$

for $i=1, \ldots, M$. Also, $\mathcal{F}_{h M}$ is $\gamma$-Hölder continuous in $x \in \mathbb{Z}_{h}^{M}$ since $\mathcal{F}_{h}$ is $\gamma$-Hölder continuous in $x \in \mathbb{Z}_{h}^{n}$. To see this, choose $x$ and $z$ in $\mathbb{Z}_{h}^{M}$ for which $\sum_{i=1}^{M} x_{i} y^{i}$ and $\sum_{i=1}^{M} z_{i} y^{i}$ are distinct, and observe that, using (4.15) and (4.2) as necessary,

$$
\begin{aligned}
& \frac{\left|\mathcal{F}_{h M}\left(x,\left\{s_{ \pm i}\right\}_{i=1}^{M}\right)-\mathcal{F}_{h M}\left(z,\left\{s_{ \pm i}\right\}_{i=1}^{M}\right)\right|}{\|x-z\|_{2}^{\gamma}} \\
& =\frac{\mid \mathcal{F}_{h}\left(\sum_{i=1}^{M} x_{i} y^{i} / h,\left\{\left(h^{2} /\left\|y^{i}\right\|_{2}^{2}\right) s_{ \pm i}\right\}_{i=1}^{M}\right)}{-\mathcal{F}_{h}\left(\sum_{i=1}^{M} z_{i} y^{i} / h,\left\{\left(h^{2} /\left\|y^{i}\right\|_{2}^{2}\right) s_{ \pm i}\right\}_{i=1}^{M}\right) \mid} \\
& \quad \leqslant\left(x-z \|_{2}^{\gamma}\right. \\
& \leqslant(n N)^{\gamma}\left(k_{1} \sum_{i=1}^{M} \frac{h^{2}}{\left\|y^{i}\right\|_{2}^{2}}\left(\left|s_{i}\right|+\left|s_{-i}\right|\right)+k_{2}\right) \\
& \leqslant n N\left(k_{1} \sum_{i=1}^{M}\left(\left|s_{i}\right|+\left|s_{-i}\right|\right)+k_{2}\right) .
\end{aligned}
$$


Finally, if $u: \mathbb{Z}_{h}^{n} \rightarrow \mathbb{R}$ is a solution of (4.1), then $u_{M}$ satisfies

$$
\mathcal{F}_{h M}\left(x, \delta^{2} u_{M}(x)\right)=0, \quad \forall x \in \mathbb{Z}_{h}^{M},
$$

and $\mathcal{F}_{h M}$ is diagonal, so that $u_{M}$ satisfies (4.13). Thus, by (4.17), (4.13) and (4.16) we have

$$
{ }_{N}[u]_{2, \gamma ; \mathbb{Z}_{h}^{n}} \leqslant C\left(n, \lambda /\left(n N^{2}\right), \Lambda, \gamma\right) n N\left(2 k_{1} n^{2} N^{2}{ }_{N}[u]_{2 ; \mathbb{Z}_{h}^{n}}+k_{2}\right),
$$

which is (4.3).

The global interpolation inequality

$$
{ }_{N}[u]_{2 ; \mathbb{Z}_{h}^{n}} \leqslant C\left(h_{0}, \varepsilon, N, n, \gamma\right)|u|_{0 ; \mathbb{Z}_{h}^{n}}+\varepsilon_{N}[u]_{2, \gamma ; \mathbb{Z}_{h}^{n}},
$$

where $0<h \leqslant h_{0}$, is easily deduced from the corresponding interior interpolation inequality [6, Lemma 5.2], so that, provided each semi-norm is finite, we may deduce (4.4) from (4.3).

Acknowledgements. While this work was carried out, the author was supported by an Australian Postgraduate Research Award and this work constitutes part of the author's PhD dissertation written under the supervision of Professor Neil S. Trudinger at the Australian National University. I acknowledge the assistance of Maciej Kocan, Peter Leviton and Neil Trudinger, and express my gratitude to a very thorough referee for his or her painstaking effort.

\section{References}

1. C. DeBoor, How small can one make the derivatives of an interpolating function?, $J$. Approx. Theory 13 (1975), 105-116.

2. J. Favard, Sur l'interpolation, J. Math. Pures Appl. 19 (1940), 281-300.

3. D. Gilbarg AND N. S. TRUdinger, Elliptic partial differential equations of second order, 2nd edn (Springer, Berlin, 1983).

4. W. Hackbusch, On the regularity of difference schemes, Part II, Regularity estimates for linear and nonlinear problems, Arkiv för Matematik 21 (1983), 3-28.

5. D. W. Holtby, Higher-order estimates for fully nonlinear difference equations, PhD thesis, Australian National University, 1996.

6. D. W. Holtby, Higher-order estimates for fully nonlinear difference equations, I, Proc. Edinb. Math. Soc. 43 (2000), 485-510.

7. T. KunkLE, A multivariate interpolant with $n$th derivative not much larger than necessary, PhD thesis, University of Wisconsin, Madison, 1991.

8. T. KUNKLE, Lagrange interpolation on a lattice: bounding derivatives by divided differences, J. Approx. Theory 71 (1992), 94-103.

9. H. J. Kuo And N. S. Trudinger, Linear elliptic difference inequalities with random coefficients, Math. Comput. 55 (1990), 37-53.

10. H. J. Kuo AND N. S. TRudinger, Discrete methods for fully nonlinear elliptic equations, SIAM J. Numer. Analysis 29 (1992), 123-135.

11. M. V. SAFONOV, On the classical solution of nonlinear elliptic equations of second order Izv. Akad. Nauk SSSR, Ser. Mat. 52 (1988), 1272-1287. (English transl.: Math. USSR Izvestiya 33 (1989), 597-612.)

12. M. V. SAfONOV, Nonlinear elliptic equations of second order, Lecture notes Università di Firenze, 1991. 